\title{
Gas-phase Reactions of Charged Phenyl Radicals with Neutral Biomolecules Evaporated by Laser-induced Acoustic Desorption
}

\author{
Christopher J. Petzold, Luis E. Ramírez-Arizmendi, Jenny L. Heidbrink, \\ James Pérez, and Hilkka I. Kenttämaa \\ Department of Chemistry, Purdue University, West Lafayette, Indiana, USA
}

\begin{abstract}
A generally applicable method for the study of phenyl radicals' reactions with neutral biomolecules in the gas phase is demonstrated. Neutral biomolecules were evaporated into a Fourier-transform ion cyclotron resonance mass spectrometer (FT-ICR) by means of laserinduced acoustic desorption (LIAD) and subsequently reacted with trapped charged phenyl radicals. The structural integrity of the evaporated alanylalanine molecules was verified by reaction with dichlorophosphenium ions. Examination of the reactions of charged phenyl radicals with alanylalanine and thymidine evaporated via LIAD revealed hydrogen atom abstraction for both alanylalanine and thymidine as well as an addition/elimination product for the reaction with thymidine. These reactions are consistent with the results obtained by others in solution. Further, a previously unstudied reaction of the nucleotide of thymine (T1) with charged phenyl radical was found to yield analogous products as the reaction with thymidine. (J Am Soc Mass Spectrom 2002, 13, 192-194) @ 2002 American Society for Mass Spectrometry
\end{abstract}

$\mathrm{R}$ adical-induced degradation of proteins and nucleic acids is thought to be the cause of various diseases [1]. The damaging effects of hydroxyl radicals are well documented [2]. However, there is also evidence that aromatic $\sigma$-radicals can damage biological molecules [3]. Thus, efforts have been directed towards understanding the interactions of phenyl radicals with biomolecules [4-6]. Unfortunately, investigation of these reactions at the molecular level is a daunting endeavor because of the highly reactive nature of the radicals and the complexity of the biological environment. Gas-phase experiments provide a tool for the examination of many reactive intermediates under clean conditions. Affixing a charged moiety to a radical permits its mass spectrometric manipulation and analysis. If an inert charged group is employed, only reactions involving the radical moiety are observed [7]. In this communication, we demonstrate the success of this experimental approach for studies of radical reactions involving gas-phase biomolecules.

Many reagents that probe the fundamental reactivity of phenyl radicals are readily introduced into the high

Published online December 6, 2001

Address reprint requests to Dr. H. I. Kenttämaa, Department of Chemistry, Purdue University, 1393 Brown Building, West Lafayette, IN 47907-1393, USA. E-mail: hilkka@purdue.edu vacuum environment of mass spectrometers [7]. However, most biological molecules, including peptides and nucleosides, are nonvolatile, thermally labile compounds that decompose upon heating before evaporation occurs. Commonly used laser-based techniques for desorption of neutral molecules, such as direct laser desorption (LD) and matrix/assisted laser desorption (MALD), impart large velocities to the desorbed molecules [8] which results in a higher percentage of hardsphere collisions and consequently lower efficiency for capture collisions necessary for most ion-molecule reactions. It is important to note that techniques which are commonly used to analyze biological molecules via mass spectrometry (i.e., matrix-assisted laser desorption/ionization (MALDI) [9] and electrospray ionization (ESI) [10]), are not applicable in these types of studies because they evaporate ionic species. As an effort to enable gas-phase studies of biomolecules, we decided to implement an alternate technique, laserinduced acoustic desorption (LIAD), for evaporation of nonvolatile neutral compounds into a Fourier-transform ion cyclotron resonance (FT-ICR) mass spectrometer [11, 12]. All experiments were carried out using a dual-cell FT-ICR mass spectrometer equipped with a specially designed probe assembly [13]. LIAD [11, 14, 15] was effected by the irradiation of a thin $(10 \mu \mathrm{m})$ copper or titanium foil with a high-intensity laser pulse 


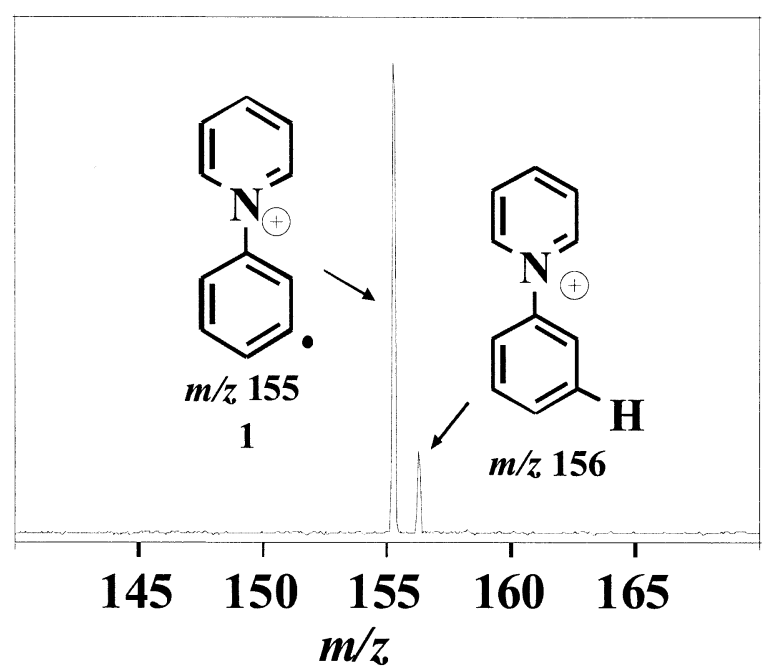

Figure 1. Mass spectrum for the reaction of the N-(3-dehydrophenyl)pyridinium ion $\mathbf{1}$ with Ala-ala evaporated via LIAD.

from a Nd:YAG laser ( 3 ns pulsewidth, $532 \mathrm{~nm}$ wavelength, fluence of $1 \times 10^{9} \mathrm{~W} \mathrm{~cm}^{-2}$ at the metal surface). The ablation of the metal by the laser pulse generates a large amplitude acoustic wave (shockwave) that propagates through the metal foil [16]. Upon reaching the opposite surface of the foil, the planar acoustic wave front gently effects the desorption of nonvolatile molecules from the surface of the metal over an area analogous to the spot size of the laser pulse [16]. By effecting LIAD over a total area of $\sim 10^{-2} \mathrm{~cm}^{2}$ (total evaporation of $\sim 100$ pmols of material), a sufficient quantity of analyte molecules can be volatilized for an observable reaction with trapped, charged phenyl radicals. The radicals were generated in one side of the dual cell by using previously published procedures [7] and transferred into the reaction cell before desorption of the neutral biomolecules into this cell.

In order to probe the validity of our method, alanylalanine (Ala-ala) evaporated via LIAD was allowed to react with the $\mathrm{N}$-(3-dehydrophenyl)pyridinium ion (1) in the gas phase. Hydrogen atom abstraction $(\mathrm{m} / \mathrm{z} 156)$ was the only reaction observed for Ala-ala (Figure 1). Based on literature results on related reactions [17], this was the expected result. However, the structural integrity of the Ala-ala molecules evaporated via LIAD was not established by this experiment. Furthermore, ion 1 reacts with the major thermal decomposition product of Ala-ala, Ala-ala anhydride, to yield the same product. Consequently, it was necessary to probe the structural integrity of Ala-ala by an alternate reaction.

Our previous findings on the reactions of phosphenium ions with various organic molecules, including small peptides [18, 19], led us to examine the reactions of Ala-ala and Ala-ala anhydride with the dichlorophosphenium ion $\left({ }^{35} \mathrm{ClP}^{37} \mathrm{Cl}, \mathrm{m} / \mathrm{z} 103\right.$, formed by electron ionization of $\mathrm{PCl}_{3}$ ). Noticeable differences are apparent in the spectra shown in Figure 2. The large signals seen at $\mathrm{m} / \mathrm{z} 143$ and 161 in Figures $2 \mathrm{a}$ and $\mathrm{b}$
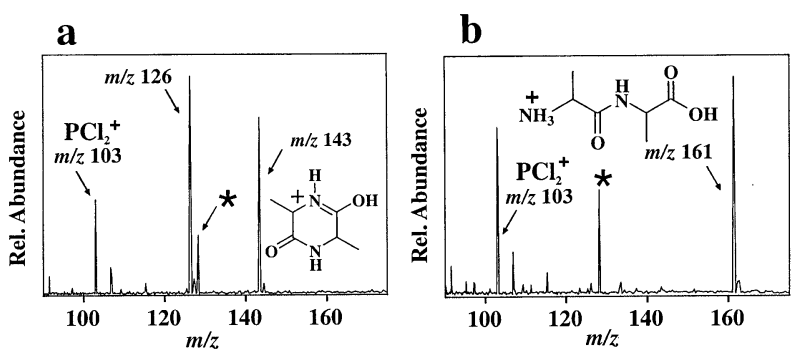

Figure 2. Mass spectra measured for reactions of $\mathrm{PCl}_{2}{ }^{+}$with (a) Ala-ala anhydride and (b) Ala-ala evaporated via LIAD. Asterisk signifies electronic noise.

correspond to protonated Ala-ala anhydride and intact protonated Ala-ala, respectively. Reduction of the amount of evaporated sample established that these ions are secondary products resulting from proton transfer to neutral Ala-ala anhydride and Ala-ala from the corresponding radical cations, $(\mathrm{m} / \mathrm{z} 142$ and 160, respectively), formed by electron abstraction by the $\mathrm{PCl}_{2}{ }^{+}$ion (IE $\sim 8.5 \mathrm{eV} \mathrm{[20]).}$

However, the most abundant reaction product of Ala-ala anhydride, an ion of $\mathrm{m} / \mathrm{z} 126$ formed by $\mathrm{O}$ anion abstraction by $\mathrm{PCl}_{2}{ }^{+}$, is not present in the spectrum of Ala-ala. We conclude that degradation of Ala-ala due to the desorption process is not occurring to a measurable degree. Hence, the $\mathrm{H}$ atom abstraction product discussed above indeed arises from the reaction of intact Ala-ala with 1. This result is in agreement with the reactivity reported earlier for two other electrophilic radicals, the hydroxyl [17] and alkoxyl [21] radicals, toward Ala-ala in solution.

Encouraged by the above results, we proceeded to examine the gas-phase reactions of two other, even more electrophilic phenyl radicals, 2 (see Scheme 1) and the $\mathrm{N}$-(4-dehydrotetrafluoro)pyridinium ion (not shown). Radical 2 reacts with Ala-ala in the same manner as 1, with hydrogen atom abstraction being the exclusive reaction channel. The same applies to reactions of these two radicals with the tetrapeptide Valala-ala-phe. The tetrafluorinated radical, however, appears to react differently. Reaction of this radical with Ala-ala occurs not only by $\mathrm{H}$ atom abstraction $(43 \%)$ but

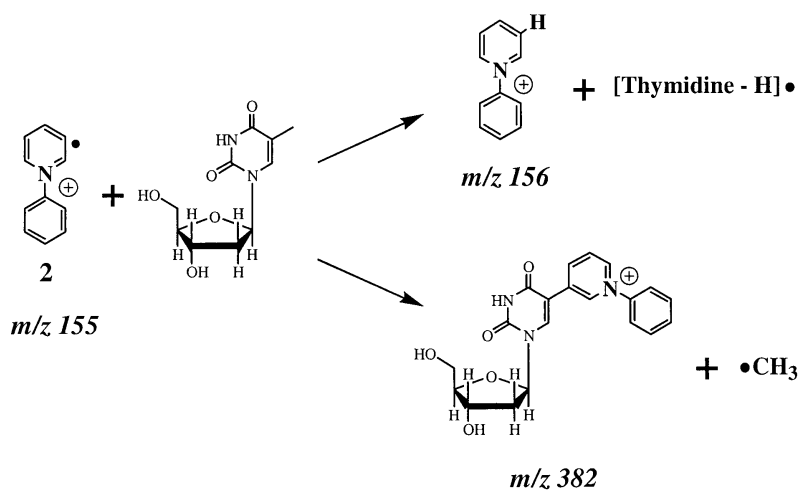

Scheme 1 


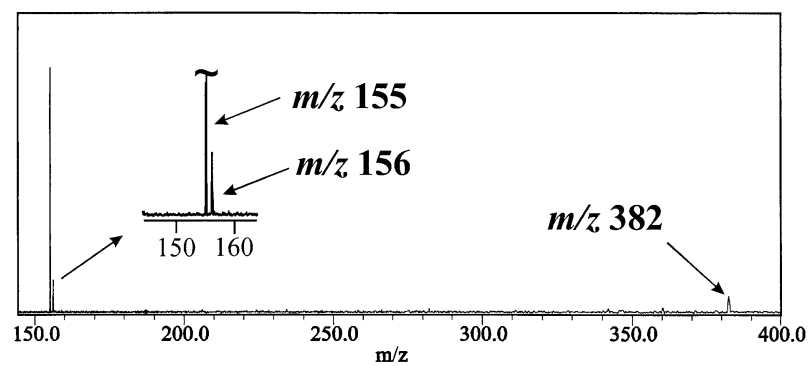

Figure 3. Mass spectrum for the reaction of the N-(phenyl)-3dehydropyridinium ion 2 with LIAD evaporated thymidine. The insert shows an expanded view of the peaks corresponding to the reactant radical and the hydrogen atom abstraction product.

also by $\mathrm{NH}_{2}$ abstraction (57\%). Hence, the substitution on the radical appears to have a major influence on its reactivity toward peptides. This issue will be addressed in our future work.

Studies on gas-phase reactions of phenyl radicals with components of nucleic acids were initiated by probing the reaction of the N-phenyl-3-dehydropyridinium ion (2) with the nucleoside thymidine. Recent solution studies [3] suggest that the phenyl radical reacts with thymidine by hydrogen atom abstraction from the sugar or nucleobase moieties, and by addition to the double bond of the nucleobase moiety. As shown in Scheme 1, reactions of $\mathbf{2}$ with gaseous thymidine, evaporated via careful thermal heating, yield analogous products, the ions corresponding to hydrogen atom abstraction $(m / z 156)$ and addition of the phenyl radical to the double bond of the base moiety, accompanied by elimination of a methyl radical $(\mathrm{m} / \mathrm{z} 382)$ [22]. Upon more vigorous heating, thermal degradation of the thymidine was revealed by the appearance of an ionic product of $\mathrm{m} / \mathrm{z} 266$ that arises from addition/methyl elimination reaction of 2 with thymine, a fragment of thymidine [22]. Thus, this reaction provides a test for the structural integrity of thymidine brought into the gas phase by LIAD. Figure 3 depicts the results obtained upon examination of the reaction of $\mathbf{2}$ with thymidine evaporated via LIAD. The ions of $m / z 156$ (hydrogen abstraction) and $m / z 382$ (addition followed by elimination of a methyl radical) were observed. The absence of the ion of $\mathrm{m} / \mathrm{z} 266$ indicates that thymidine did not undergo degradation during the LIAD process. Furthermore, the branching ratios of hydrogen abstraction to addition/elimination measured in the low-heat thermal evaporation and LIAD experiments for this nucleoside are nearly identical (37:63 and 34:66, respectively, for hydrogen abstraction:addition/methyl elimination).

Gas-phase experiments on neutral nucleotides have been hindered by the extensive degradation of nucleotides upon thermal heating. However, LIAD enables studies of radical reactions of nucleotides in the gas phase. We demonstrated this by examining the reaction of 2 with the nucleotide thymidylic acid (T1), desorbed by LIAD (thermal heating led to complete degradation of the sample). Analogous to the reaction of thymidine, hydrogen atom abstraction and addition accompanied by loss of a methyl radical $(\mathrm{m} / \mathrm{z} 463)$ were observed.

As a conclusion, we have demonstrated a feasible experimental approach for the study of radical reactions of biomolecules in the gas phase. This approach allowed us to identify reaction products and obtain semi-quantitative information on relative reaction rates for interaction of a substituted phenyl radical with nucleotides and a nucleotide, as well as small peptides. This experimental approach will be applied in our future work to delineate structure/reactivity relationships for estimation of organic radicals' ability to damage oligopeptides and oligonucleotides.

\section{Acknowledgments}

The authors wish to thank Jason M. Price for helpful discussions. Financial support was provided by the National Institutes of Health. CJP is grateful to Pfizer for a predoctoral fellowship.

\section{References}

1. Dean, R. T.; Fu, S.; Stocker, R.; Davies, M. J. Biochem. J. 1997, 324,1 .

2. Blackburn, G. M.; Gait, M. J. IRL Press: Oxford, 1990.

3. Hazelwood, C.; Davies, M. J.; Gilbert, B. C.; Packer, J. E. J. Chem. Soc. Perkin Trans. 2 1995, 2167.

4. Akman, S. A.; Doroshow, J. H.; Kensler, T. W. Carcinogenesis 1992, 13, 1783.

5. Greenley, T. L.; Davies, M. J. Biochem. Biophys. Acta 1993, 1157, 23.

6. Braslau, R.; Anderson, M. O. Tetrahedron Lett. 1998, 39, 4227.

7. Smith, R. L.; Kenttämaa, H. I. J. Am. Chem. Soc. 1995, 117, 1393.

8. Miller, J. C.; Haglund, R. F. Academic: San Diego, 1998.

9. Karas, M.; Bachmann, D.; Bahr, U.; Hillenkamp, F. Int. J. Mass Spectrom. Ion Processes 1987, 78, 53.

10. Fenn, J. B.; Man, C. K.; Meng, C. K.; Wong, S. F. Mass Spectrom. Rev. 1990, 9, 37.

11. Pérez, J.; Ramírez-Arizmendi, L. E.; Petzold, C. J.; Guler, L. P.; Nelson, E. D.; Kenttämaa, H. I. Int. J. Mass Spectrom. 2000, 198, 173.

12. Reid, G. E.; Tichy, S. E.; Pérez, J.; O’Hair, R. A. J.; Simpson, R. J.; Kenttämaa, H. I. J. Am. Chem. Soc. 2001, 123, 1184.

13. Pérez, J.; Petzold, C. J.; Watkins, M. A.; Vaughn, W. E.; Kenttämaa, H. I. J. Am. Soc. Mass Spectrom. 1999, 10, 1105.

14. Golovlev, V. V.; Allman, S. L.; Garrett, W. R.; Taranenko, N. I.; Chen, C. H. Int. J. Mass Spectrom. Ion Processes 1997, 169/170, 69.

15. Lindner, B. Int. J. Mass Spectrom. Ion Processes 1991, 103, 203.

16. Scruby, C. B.; Drain, L. E. Laser Ultrasonics. Adam Hilger Press: New York, 1990.

17. Hawkins, C. L.; Davies, M. J. Chem. Soc. Perkins Trans. 2 1998, 1937.

18. Ramírez-Arizmendi, L. E.; Yu, Y.-Q.; Kenttämaa, H. I. J. Am. Soc. Mass Spectrom. 1999, 10, 379.

19. Yu, Y.-Q.; Stumpf, C. L.; Kenttämaa, H. I. Int. J. Mass Spectrom. 2000, 195/196, 609.

20. Brum, J. L.; Hudgens, J. W. J. Phys. Chem. 1994, 98, 5587.

21. Headlam, H. A.; Mortimer, A.; Easton, C. J.; Davies, M. J. Chem. Res. Toxicol. 2000, 13, 1087.

22. Ramírez-Arizmendi, L. E.; Heidbrink, J. L.; Guler, L. P.; Kenttämaa, H. I. in preparation. 